\title{
EL ÚLTIMO CONCIERTO (2012): LA FISIOTERAPIA EN ENFERMEDAD DE PARKINSON PARA DESACELERAR LA CAÍDA DE UNA ESTRELLA DE LA MÚSICA CLÁSICA
}

\section{A Late Quartet (2012): Physical therapy for Parkinson's disease as a way to slow the fall of a classical music star}

Montserrat GUILLERMO-DURÁN, Ileana CÁMARA-OROPEZA, Nina MÉNDEZ-DOMÍNGUEZ

Universidad Marista de Mérida, Campus de Ciencias de la Salud (México).

Autor para correspondencia: Nina Méndez Domínguez.

Correo electrónico: ninuxka@hotmail.com

Recibido: 12 de agosto de 2020

Aceptado: 21 de agosto de 2020

\section{Resumen}

El último concierto/ A Late Quartet (2012) es una película del género dramático dirigida por Yaron Zilberman. Este filme protagonizado por Christopher Walken, relata la historia de un cuarteto de cuerda, el cuál tras 25 años de éxito y fama mundial, atraviesa fuertes adversidades cuando el creador del cuarteto, Peter Mitchell, está padeciendo los primeros síntomas del Párkinson, una enfermedad que en poco tiempo pondrá fin a su pasión y a sus sueños como intérprete. Pero ¿qué hubiera podido ofrecerse para el manejo de Peter desde la fisioterapia y rehabilitación? ¿EI impacto de esta profesión en la calidad de vida, pudiera ayudar a mitigar la cuesta debajo de un gran músico con enfermedad de Párkinson que se ubica en la cima de su carrera?

El objetivo del presente artículo es describir las alternativas basadas en evidencia que la fisioterapia y rehabilitación pueden ofrecer a los pacientes con enfermedad de Párkinson.

Palabras clave: enfermedad de Parkinson; fisioterapia; enfermedades neurodegenerativas.

\section{Abstract}

A Late Quartet (2012) is a film of the dramatic genre directed by Yaron Zilberman. This film, starring Christopher Walken, provides an insight to the story of a string quartet, which after 25 years of success and world fame, goes through severe adversities when the creator of the quartet, 


\author{
EL ÚLTIMO CONCIERTO (2012): LA FISIOTERAPIA EN ENFERMEDAD DE PARKINSON \\ PARA DESACELERAR LA CAÍDA DE UNA ESTRELLA DE LA MÚSICA CLÁSICA \\ MONTSERRAT GUILLERMO-DURÁN, ILEANA CÁMARA-OROPEZA, NINA MÉNDEZ-DOMÍNGUEZ
}

\begin{abstract}
Peter Mitchell, is suffering from the first symptoms of Parkinson's Disease, a disease that threatens to end to his passion and his dreams as a performer. But what could have been offered for Peter's management from physical therapy specialty for rehabilitation? Could it provide a positive impact for this profession on his quality of life? Could it help mitigate the downhill slope of a great musician with Parkinson's disease from the peak of his career?

The objective of this article is to describe the evidence-based alternatives that physical therapy and rehabilitation can offer to patients with Parkinson's disease.
\end{abstract}

Key Words: Parkinson disease; physical therapy specialty; neurodegenerative diseases.

Ficha técnica

Título: El último concierto.

Título original: A Late Quartet.

País: Estados Unidos.

Año: 2012.

Director: Yaron Zilberman.

Música: Angelo Badalamenti.

Fotografía: Frederick Elmes.

Montaje: Frederick Elmes

Guion: Seth Grossman, Yaron Zilberman.

Intérpretes: Philip Seymour Hoffman, Catherine Keener, Christopher Walken, Mark Ivanir, Imogen Poots, Madhur Jaffrey, Liraz Charhi, Wallace Shawn, Pamela Quinn, Brooklyn Parkinson Group, Cristian Puig, Rebeca Tomas, Megan McQuillan, David Redden, Ted Hartley, ...

Color: color.

Duración: 105 minutos.

Género: drama; música; enfermedad; amistad.

Idioma original: inglés.

Sinopsis: «Un cuarteto de cuerda de Nueva York atraviesa dificultades cuando el violonchelista de la formación presenta síntomas iniciales de la enfermedad de Parkinson» (Filmaffinity)

Productora: Opening Night Productions, Concept Entertainment, Unison Films, Spring Pictures, RKO Pictures.

\section{Enlaces:}

https: / / w w w.imd b.com/title / tt1226240/?ref_=nv_sr_1?ref=nv_sr_1 https://www.filmaffinity.com/es/film385437. html

\section{Introducción}

El último concierto / A Late Quartet (2012) de Yaron Zilberman, nos ofrece una mirada profunda hacia la vida de quienes padecen la enfermedad de Parkinson, nos permite reflexionar qué tanto puede cambiar el curso de una vida. A través de un filme dramático Zilberman nos muestra la historia de Peter Mitchell, un músico que forma parte de un famoso cuarteto de cuerdas de Nueva York que se encuentra en su mejor momento y preparándose para celebrar la temporada que conmemora los 25 años de su conformación.

La trama comienza cuando Peter, un chelista impecable y de renombre, experimenta dificultades para controlar la precisión de sus dedos al tocar el chelo, más tarde se enteraría que dichas manifestaciones eran los síntomas iniciales de un trastorno neurodegenerativo (Foto 1). El diagnóstico de Peter no resultó difícil al ojo clínico del especialista, pues ya mostraba datos de hipocinesia lateral, marcha atípica y dificultad para mantener el equilibrio, todo ello característico de la enfermedad de Parkinson.

Es así, como un famoso músico en la cima del éxito ha de enfrentar un duro descenso para enfrentar una vida con limitaciones físicas que le impedirán en gran manera expresar su enorme talento. La enfermedad de Parkinson es un padecimiento que ocurre cuando las células nerviosas no producen suficiente cantidad de dopamina, una importante sustancia química implicada en el control del movimiento.

\title{
Trailer en español
}




\section{EL ÚLTIMO CONCIERTO (2012): LA FISIOTERAPIA EN ENFERMEDAD DE PARKINSON \\ PARA DESACELERAR LA CAIIDA DE UNA ESTRELLA DE LA MÚSICA CLÁSICA}

MONTSERRAT GUILLERMO-DURÁN, ILEANA CÁMARA-OROPEZA, NINA MÉNDEZ-DOMÍNGUEZ

El último concierto presenta una conmovedora historia de lucha y de retos, que le permite al público comprender la evolución de un padecimiento, que a pesar de los múltiples esfuerzos por encontrar un tratamiento que remita los síntomas, continúa sin tener cura, pero ello no significa que no existan alternativas que puedan aminorar las manifestaciones clínicas de la enfermedad y permitir una mejor calidad de vida a quienes la padecen; en este sentido la fisioterapia y rehabilitación dentro del manejo integral del paciente con Parkinson son fundamentales por los motivos que expondremos a continuación. Debido a ello, el objetivo del presente artículo es describir las terapias complementarias basadas en evidencia que la fisioterapia y rehabilitación pueden ofrecer a los pacientes con enfermedad de Parkinson.

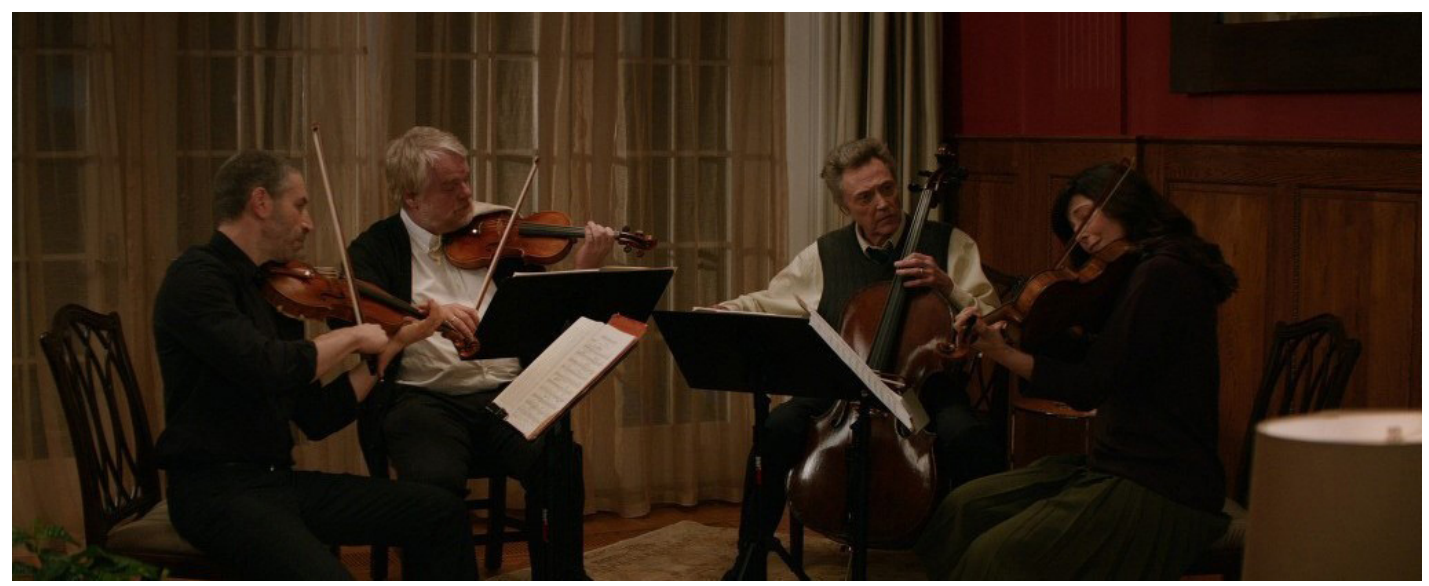

Foto 1. El cuarteto se encuentra preparando su próximo concierto, Peter experimenta dificultades para tocar el Chelo.

\section{Enfermedad de Parkinson}

El término parkinsonismo agrupa al conjunto de enfermedades que se caracterizan por una alteración del control del movimiento. La enfermedad de Parkinson (EP) ha sido reconocida como la entidad clínica más frecuente dentro de los casos del síndrome general del parkinsonismo. Por otra parte. se encuentra el parkinsonismo secundario, término que hace referencia a aquellas patologías que cursan con un cuadro clínico similar al de la EP pero cuya etiología se asocia con la ingesta de fármacos, con traumatismos, así como vasculopatías ${ }^{1,2}$.

Debe destacarse que la enfermedad de Parkinson ha sido reconocida como la segunda enfermedad neurodegenerativa más común a nivel mundial. Se estima que para el 2040 se convertirá en la enfermedad grave más común con más 14 millones de personas afectadas en el mundo ${ }^{3,4}$. En los países industrializados, la prevalencia estimada es de $0.3 \%$ en la población general, $1.0 \%$ en personas mayores de 60 años y $3.0 \%$ en personas mayores de 80 años. Se ha observado que la incidencia varía entre 8 y 18 por cada 100000 persona-años ${ }^{5}$. Tanto la incidencia como la prevalencia son de 1,5 a 2 veces mayores en hombres que en mujeres, lo que ha sugerido un posible efecto protector de los estrógenos. Por otra parte, se han observado diferencias interétnicas que 


\section{EL ÚLTIMO CONCIERTO (2012): LA FISIOTERAPIA EN ENFERMEDAD DE PARKINSON \\ PARA DESACELERAR LA CAIIDA DE UNA ESTRELLA DE LA MÚSICA CLÁSICA \\ MONTSERRAT GUILLERMO-DURÁN, ILEANA CÁMARA-OROPEZA, NINA MÉNDEZ-DOMÍNGUEZ}

exponen un mayor riesgo de incidencia en poblaciones hispanas 6,7 .

Fisiopatológicamente, se caracteriza por la pérdida de neuronas dopaminérgicas en la sustancia negra y la presencia de inclusiones llamadas cuerpos de Lewy. Se ha descrito que, al momento de presentarse los primeros síntomas, la mayoría de la vía dopaminérgica ya se encuentra degenerada, lo que explica porque es una patología difícil de abordar ${ }^{1,3}$.

Para diversos especialistas de las ciencias básicas y clínicas significa aún un reto determinar el origen de este padecimiento, cuya génesis se ha identificado al menos parcialmente. A pesar de que se conocen los mecanismos fisiopatológicos implicados, el origen continúa siendo incierto. Actualmente se apoya una etiología multifactorial ${ }^{9}$,

${ }^{10}$. Con respecto a los factores de riesgo, la edad se considera el principal de éstos, existe un pico de prevalencia entre los 85 y 89 años. En segunda instancia, se encuentra la historia familiar y finalmente los factores ambientales, como es el caso de la exposición a tóxicos ambientales entre los cuales destacan algunos pesticidas de uso habitual como el paraquat ${ }^{1,9}$.

\section{Cuadro clínico de la enfermedad de Parkinson}

Los síntomas motores cardinales de la EP son temblor, rigidez, bradicinesia (acinesia) e inestabilidad postural; en el caso del protagonista es posible apreciar los primeros tres síntomas antes mencionados, ya que son los que caracterizan el cuadro clínico inicial. Cabe destacar que la EP también incluye síntomas no motores, los cuales predominan en la fase avanzada de este padecimiento. Dentro de éstos se encuentran los trastornos neuropsiquiátricos como la depresión, la ansiedad y la apatía; trastornos cognitivos como la demencia, así como trastornos gastrointestinales, genitourinarias y alteraciones del sueño ${ }^{1}$. La trama de «El último concierto» permite notar que la enfermedad se manifiesta con cambios degenerativos progresivos que incluyen manifestaciones motoras, Peter comienza a experimentar trastornos neuropsiquiátricos como la depresión y la apatía.

La escala de Hoehn y Yahr, se ha convertido en el estándar de oro para estimar la gravedad de la EP, estableciendo cinco estadios de severidad dependiendo del nivel de afectación motora, partiendo desde la presencia de la disfunción general del parkinsonismo hasta una afectación motora bilateral que compromete la marcha y el equilibrio ${ }^{11}$. En el caso del Peter, se encuentra en el primer estadio de evolución, de modo que los temblores y las manifestaciones motoras se observan únicamente en un hemisferio corporal y no presenta graves alteraciones de la postura y de la marcha.

\section{Diagnóstico clínico}

El diagnóstico de esta enfermedad es esencialmente clínico. Dado que no existe una prueba que permita confirmarlo, se han desarrollado diferentes criterios diagnósticos. La actualización más reciente fue realizada por la Sociedad de Trastornos del Movimiento, los cuales cuentan con una precisión diagnóstica del $92 \%$, sensibilidad del $94.5 \%$ y especificidad del $88.5 \%^{1,12}$. De hecho, en el film podemos observar el momento del diagnóstico, que no le llevó más que una observación dirigida a la especialista para poder diagnosticar la enfermedad de Parkinson, pues ésta suele evidenciarse cuando el paciente realiza movimientos, se desplaza o incluso, cuando se comunica. Sin embargo, dada la complejidad de la EP y los múltiples diagnósticos diferenciales, es importante aplicar instrumentos de valoración específicos para poder confirmarlo (Foto 2).

Existen también las guías de fisioterapia que establecen las pautas del proceso diagnóstico de la EP en rehabilitación a la vez que permiten llevar monitoreo del grado de progresión de la enfermedad. La anamnesis de fisioterapia debe comenzar por el conocimiento de los problemas percibidos por el paciente, así como la evolución de la 


\section{EL ÚLTIMO CONCIERTO (2012): LA FISIOTERAPIA EN ENFERMEDAD DE PARKINSON \\ PARA DESACELERAR LA CAIIDA DE UNA ESTRELLA DE LA MÚSICA CLÁSICA}

MONTSERRAT GUILLERMO-DURÁN, ILEANA CÁMARA-OROPEZA, NINA MÉNDEZ-DOMÍNGUEZ

enfermedad y el estado actual. Existen diferentes escalas de medición con resultados estandarizados, que pueden emplearse como instrumentos para conocer tanto el estado de salud general como el estado de salud en áreas específicas ${ }^{13}$. Una buena evaluación clínica facilita la comprensión del paciente sobre su padecimiento y garantiza una mejor adherencia terapéutica. Es por ello que, actualmente se sugiere realizar una valoración integral de estos pacientes, que contemple a la fisioterapia como parte de este proceso.

Un momento crucial en la historia del músico se representa en la escena en la cual Peter acude al médico, quien, tras aplicar algunas pruebas motoras, con poca certeza diagnóstica determina que probablemente Peter presentaba enfermedad de Parkinson, a lo que éste responde "Bueno, pero...con esto que acabamos de hacer ¿puede darse cuenta de que tengo Parkinson?». Es interesante cuestionar la importancia de una adecuada evaluación clínica, puesto que el objetivo de ésta no se limita a determinar un diagnóstico, sino conocer las condiciones de salud del paciente, que serán la pauta para establecer los objetivos terapéuticos y diseñar un tratamiento personalizado.

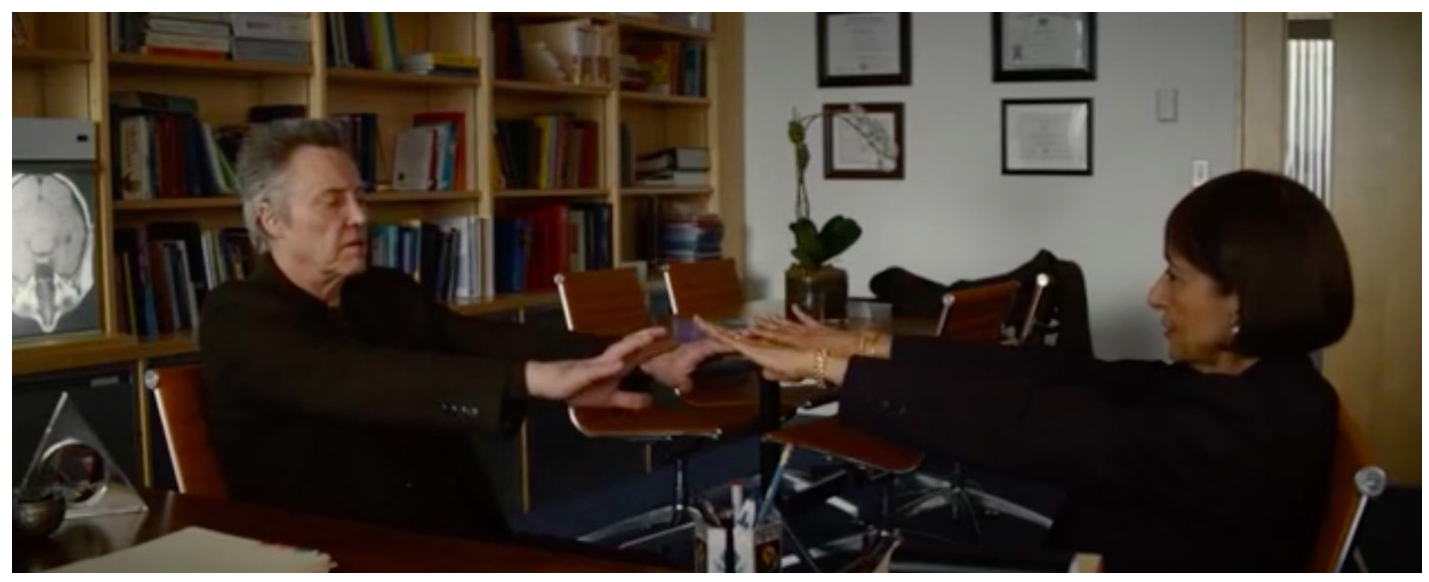

Foto 2. Evaluación clínica realizada por el neurólogo.

Aunque el hecho de transmitir un diagnóstico pueda parecer algo sencillo, el profesional de la salud no debe olvidar que la empatía, el respeto y la comprensión, son elementos fundamentales de la relación médico-paciente y es posiblemente por el modo en el cual se le comunicó su diagnóstico que Peter no puede más que expresar una cierta sorpresa al enterarse.

\section{Tratamiento farmacológico}

El tratamiento de la EP es predominantemente sintomático. Hasta la fecha no existe evidencia de un tratamiento modificador de la enfermedad ni tampoco de fármacos neuroprotectores. La levodopa ha sido reconocida como el estándar de oro, ya que es el fármaco más efectivo para el tratamiento de los síntomas motores. Sin embargo, está asociado con el desarrollo de graves complicaciones discapacitantes como fluctuaciones, discinesias y distonías que empeoran el pronóstico de estos pacientes ${ }^{1,14}$.

Los profesionales de la salud deben informar y educar al paciente sobre los beneficios y las reacciones adversas de los medicamentos. Tras 
recibir el diagnóstico, la vida de Peter da un giro de 360 o, pudiéndose apreciar cómo la falta de información y de comunicación activa con el médico puede desencadenar altos niveles de ansiedad y un panorama totalmente devastador (Foto 3).

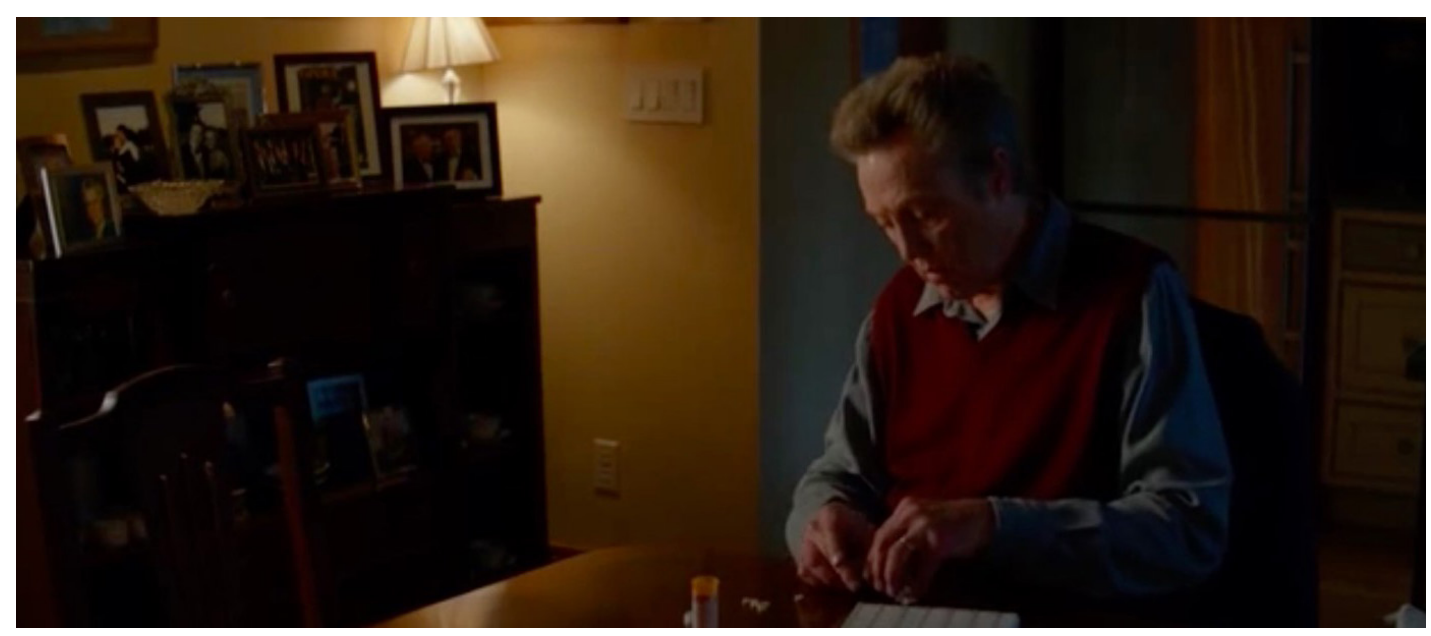

Foto 3. Peter tomando sus medicamentos, desilusionado porque el tratamiento no funciona como se esperaba.

Intervención desde la fisioterapia y rehabilitación

Los objetivos de tratamiento en fisioterapia cambian conforme la enfermedad progresa. La intensidad y la duración de las sesiones se correlacionan con los objetivos. Las áreas centrales abordadas por la fisioterapia en este padecimiento son: las capacidades físicas, las transferencias, las actividades manuales, la postura, el equilibrio y la marcha ${ }^{13}$.

La evidencia científica ha demostrado que las intervenciones fisioterapéuticas pueden ser eficaces para mejorar la sintomatología a través de diferentes enfoques de rehabilitación. Se han establecido recomendaciones y pautas sobre el tratamiento del paciente con EP. Se ha demostrado que un óptimo tratamiento rehabilitador debe contemplar cuatro pilares: la educación, el ejercicio, el entrenamiento cognitivo mediante la señalización, así como el entrenamiento estratégico ${ }^{13,15}$.
La terapia de señalización parece actuar principalmente sobre los bloqueos motores y la capacidad de mantener una adecuada amplitud motora. La falta de motivación, la fatiga y las bajas expectativas en estos pacientes, representan barreras para la realización de actividad física, sin embargo, la terapia mediante el baile se ha propuesto como una interesante alternativa de tratamiento rehabilitador ${ }^{15}$.

Debido a la creciente evidencia científica sobre los efectos de las intervenciones fisioterapéuticas, se ha transformado el abordaje de la EP. Cada vez son más, los especialistas que se inclinan por un tratamiento integral que contemple a la fisioterapia como parte de éste ${ }^{16}$. Peter recibe un tratamiento exclusivamente farmacológico, únicamente en una escena se aprecia que realiza una sesión de ejercicio aeróbico sin supervisión alguna (Foto 4). El enfoque rehabilitador debe ser un elemento que forme 


\section{EL ÚLTIMO CONCIERTO (2012): LA FISIOTERAPIA EN ENFERMEDAD DE PARKINSON \\ PARA DESACELERAR LA CAIIDA DE UNA ESTRELLA DE LA MÚSICA CLÁSICA}

MONTSERRAT GUILLERMO-DURÁN, ILEANA CÁMARA-OROPEZA, NINA MÉNDEZ-DOMÍNGUEZ

parte de la rutina del paciente, con una adecuada periodización, dosificación y supervisión. La terapia física no es una forma de mantener ocupado al paciente con Parkinson, debe ser un componente fundamental del abordaje terapéutico, ya que puede mejorar el pronóstico de estos.

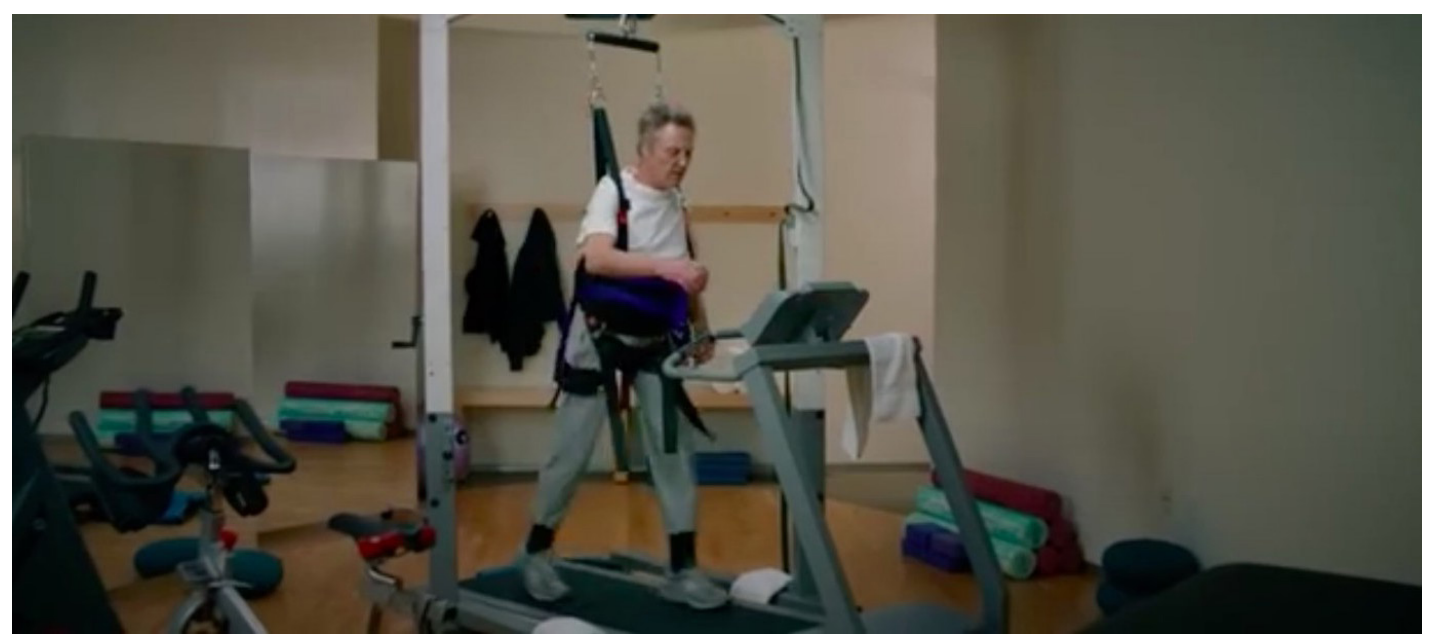

Foto 4. Peter acude a una sesión de ejercicio, la cual se realiza sin supervisión de un profesional de la salud.

Impacto de enfermedad de Parkinson en el ambiente personal

La enfermedad de Parkinson implica una serie de trastornos físicos, psicológicos, emocionales, económicos y sociales, e involucra un constante proceso de adaptación que conlleva un mayor riesgo de aislamiento social, maltrato y desvinculación de los pacientes ${ }^{17}$. Esto explica por qué Peter comenzó a distanciarse de sus amigos, convirtiéndose en una persona desmotivada que vive en un estado de tristeza profunda.

En la mayoría de los casos, ocurre una evolución de la enfermedad de entre 1 y 10 años con un mal pronóstico. Conforme la enfermedad progresa se desencadenan alteraciones de la funcionalidad que generan un importante grado de discapacidad que compromete significativamente la calidad de vida de estos pacientes ${ }^{18}$.
Es importante considerar que el dolor físico y psicológico, la desesperanza y la desconexión con el medio están presentes tras el diagnóstico de numerosos procesos neurológicos, especialmente de enfermedades neurodegenerativas, como es el caso del Parkinson. Es común que aparezca la ideación suicida en la etapa próxima al diagnóstico ${ }^{19}$. Tras recibir la noticia, el protagonista se ve inmerso en un panorama desalentador, que empeora día con día debido a la falta de una intervención integral y de redes de apoyo en su vida. Peter es viudo y prácticamente no cuenta con ningún familiar cercano; lo que llevan al protagonista a considerar la idea de suicidarse.

Impacto de enfermedad de Parkinson en el ambiente psicosocial y laboral

La enfermedad de Parkinson es un padecimiento progresivo e incapacitante, con cargas 


\section{EL ÚLTIMO CONCIERTO (2012): LA FISIOTERAPIA EN ENFERMEDAD DE PARKINSON \\ PARA DESACELERAR LA CAIIDA DE UNA ESTRELLA DE LA MÚSICA CLÁSICA}

MONTSERRAT GUILLERMO-DURÁN, ILEANA CÁMARA-OROPEZA, NINA MÉNDEZ-DOMÍNGUEZ

emocionales, económicas y sociales que no solo afectan al paciente, sino a los cuidadores y familiares de éstos. De ahí la importancia de que sean referidos a grupos y asociaciones que proporcionen información, redes de apoyo e iniciativas útiles tanto para los pacientes como sus familiares ${ }^{1,20}$. En el caso de Peter, éste es referido a un grupo de apoyo, acudió a una sesión, pero él no colaboró y prefirió retirarse sin participar (Foto 5). Es importante que los pacientes lleven un acompañamiento psicológico durante este proceso y no sean referidos únicamente a un grupo, puesto será difícil que logren acoplarse y pudiera no resultar una estrategia efectiva.

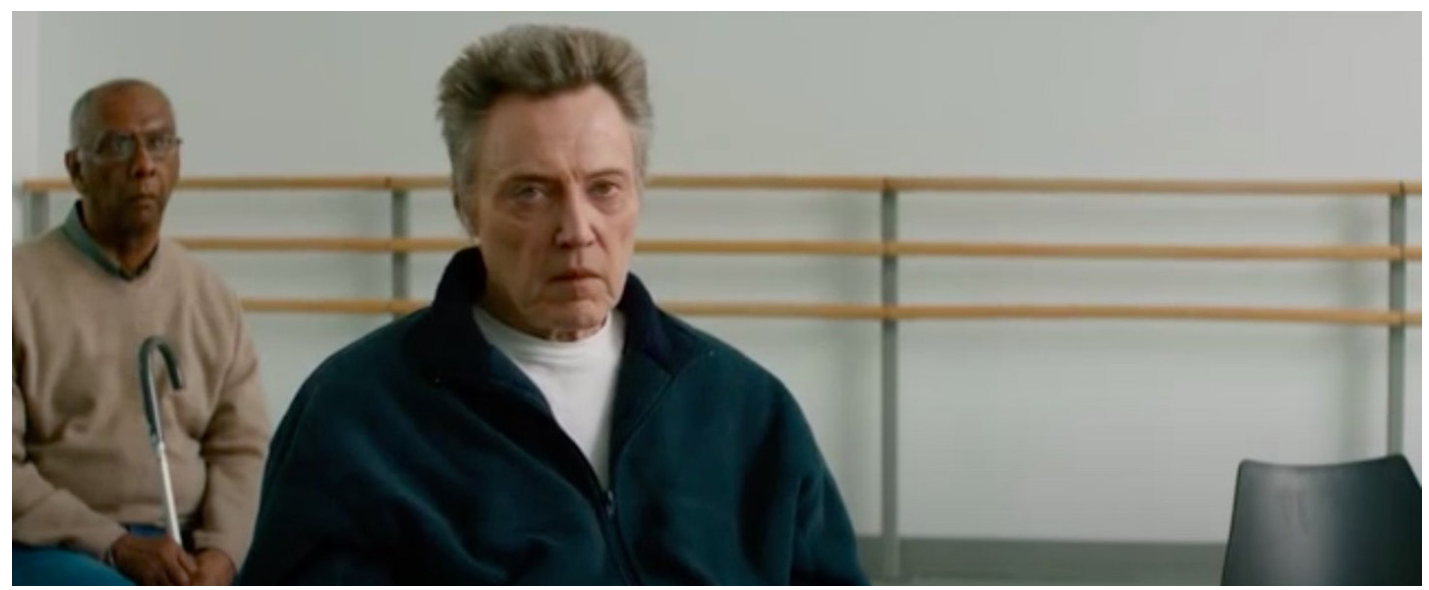

Foto 5. Peter se encuentra en estado de tristeza profunda y negación total, acude a una sesión de grupo pero no colabora y se retira sin participar.

Esta historia no sólo escenifica el impacto a nivel personal que representa un padecimiento crónico degenerativo que deteriora la calidad de vida de las personas que lo padecen y al mismo tiempo, le permite al espectador acercarse de principio a fin al entorno social y laboral de estos pacientes, reflejando las dificultades que se presentan en torno a este padecimiento.

Peter Mitchell, no sólo era un músico de renombre, representaba el núcleo y la guía que mantenía unida al cuarteto. Pronto, la incertidumbre y la ansiedad comienzan a tomar el control de su vida. Desesperado y sin ilusiones decide tomar una importante decisión que podría afectar gravemente al cuarteto, jubilarse y buscar un reemplazo para el grupo. Sin la presencia de Peter al frente del cuarteto, la envidia y las rivalidades comienzan a apoderarse del grupo generando múltiples conflictos.

Es común que algunos pacientes experimenten una mejoría significativa en los síntomas motores, tras varias semanas de ingerir los medicamentos. Es ahí cuando Peter decide regresar al cuarteto, prometiendo tocar una última vez para despedirse. Iniciada la presentación, el protagonista se da cuenta de que no es capaz de alcanzar el ritmo musical, súbitamente a la mitad del concierto deja de tocar y solemnemente se despide frente a la audiencia, dando fin a su carrera como músico (Foto 6). 


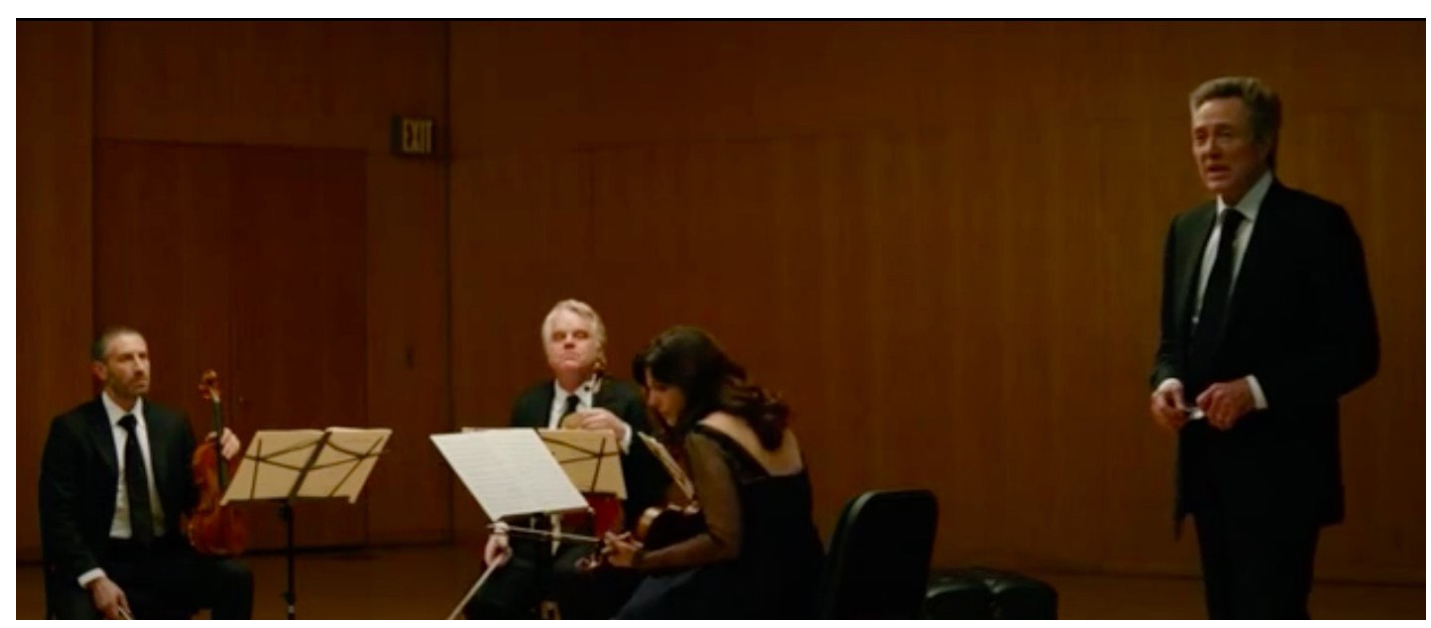

Foto 6. Peter hace público el fin de su carrera como músico, durante el último concierto.

La musicoterapia en la rehabilitación neurológica, una ventana de oportunidad en el tratamiento de la enfermedad de Parkinson

La música no sólo representa una fuente de entretenimiento y conocimiento, sino que puede convertirse en una herramienta terapéutica complementaria. Durante la última década ha aumentado el interés por utilizar la música como una estrategia en la rehabilitación neurológica. Se han desarrollado métodos de tratamiento basados en música que han demostrado mejoras significativas en déficits tanto motores, cognitivos, emocionales como sociales en personas afectadas por diversos padecimientos, dentro de los que destaca la enfermedad de Parkinson. Se ha propuesto que las redes neurales involucradas en el procesamiento musical pueden inducir cambios humorales, electrofisiológicos e incluso estructurales en el cerebro ${ }^{20}$.

Actualmente, aplicar música en la rehabilitación neurológica ha resultado ser un recurso económico con evidencia científica que respalda sus beneficios, por lo que puede ser útil en manejo de diversas condiciones médicas ${ }^{20}$. De esta forma, la música, lejos de ser un obstáculo en la vida de Peter, pudiera convertirse en una importante estrategia terapéutica, evitando que éste tenga que renunciar a sus sueños y a su mayor anhelo en la vida. Es cierto, que tal vez no podrá tocar como antes, pero es posible que ésta le ayude a mejorar su salud mientras continúa disfrutando de su pasión.

\section{Conclusión}

El último concierto / A Late Quartet (2012) nos ha permitido ver las implicaciones físicas y sociales de la enfermedad de Parkinson en lo cotidiano, en las expresiones artísticas del protagonista y en su desarrollo profesional, pero también nos llevó a revisitar la importancia de emplear los recursos de la fisioterapia basados en la evidencia que permiten ofrecer una mejor calidad de vida al paciente. Tras analizar esta trama es posible apreciar que si Peter Mitchell, hubiera recibido un tratamiento integral que incluyera un abordaje fisioterapéutico y psicológico como coadyuvantes del enfoque farmacológico, pudiera haber tenido un pronóstico más favorable y una mejora notable en la calidad de vida que retrasara su jubilación. La fisioterapia y rehabilitación busca como fin último generar un impacto positivo en la calidad 


\section{EL ÚLTIMO CONCIERTO (2012): LA FISIOTERAPIA EN ENFERMEDAD DE PARKINSON PARA DESACELERAR LA CAIIDA DE UNA ESTRELLA DE LA MÚSICA CLÁSICA \\ MONTSERRAT GUILLERMO-DURÁN, ILEANA CÁMARA-OROPEZA, NINA MÉNDEZ-DOMÍNGUEZ}

de vida de los pacientes, logrando potenciar sus capacidades para que puedan adaptarse a las nuevas circunstancias de vida.

Se puede concluir, que, siempre que los profesionales de la salud trabajan en forma conjunta, es posible implementar mejores programas de intervención terapéutica y de esta forma mejorar la calidad de vida de las personas con enfermedad de Parkinson, lo que representan un área de oportunidad para los profesionales del área de la fisioterapia y rehabilitación geriátrica.

\section{Referencias}

1. Balestrino R, Schapira AHV. Parkinson disease. Eur J Neurol. 2020;27(1):27-42.

2. Dickson DW. Parkinson's disease and parkinsonism: neuropathology. Cold Spring Harb Perspect Med. 2012;1;2(8):a009258.

3. Alim MA, Ma QL, Takeda K, Aizawa T, Matsubara M, et al. Demonstration of a role for alpha-synuclein as a functional microtubule-associated protein. J Alzheimers Dis. 2004;6(4):435-42; discussion 443-9.

4. García-Ramos R, López Valdés E, Ballesteros L, Jesús $S$, Mir P. The social impact of Parkinson's disease in Spain: Report by the Spanish Foundation for the Brain. Neurologia. 2016;31(6):401-13.

5. Poewe W, Seppi K, Tanner CM, Halliday GM, Brundin $P$, Volkmann J, et al. Parkinson disease. Nat Rev Dis Primers. 2017;23;3:17013.

6. Lee A, Gilbert RM. Epidemiology of Parkinson Disease. Neurol Clin. 2016;34(4):955-65.

7. Van Den Eeden SK, Tanner CM, Bernstein AL, Fross RD, Leimpeter A, Bloch DA, et al. Incidence of Parkinson's disease: variation by age, gender, and race/ethnicity. Am J Epidemiol. 2003; 1;157(11):1015-22.

8. Haaxma CA, Bloem BR, Borm GF, Oyen WJ, Leenders $\mathrm{KL}$, Eshuis $\mathrm{S}$, et al. Gender differences in Parkinson's disease. J Neurol Neurosurg Psychiatry. 2007;78(8):819-24.

9. Goldman SM, Marek K, Ottman R, Meng C, Comyns K, Chan P, Ma J, Marras C, Langston JW, Ross GW, Tanner CM. Concordance for Parkinson's disease in twins: A 20-year update. Ann Neurol. 2019;85(4):600-5.
10. Hurtado F, N Cárdenas MA, Cardenas F, León LA. La enfermedad de Parkinson: Etiología, tratamientos y factores preventivos. Univ Psychol. 2016;15(spe5):1-26.

11. Hoehn MM, Yahr MD. Parkinsonism: onset, progression and mortality. Neurology. 1967;17(5):427-42.

12. Postuma RB, Poewe W, Litvan I, Lewis S, Lang AE, Halliday G, et al. Validation of the MDS clinical diagnostic criteria for Parkinson's disease. Mov Disord. 2018;33(10):1601-8.

13. Keus SH, Munneke M, Graziano M, Paltamaa J, Pelosin E, Domingos J, et al. European guidelines for physiotherapy in Parkinson's disease. Movement Disorders. 2013;28:S382-3.

14. Fox SH, Katzenschlager R, Lim SY, Ravina B, Seppi $\mathrm{K}$, Coelho $\mathrm{M}$, et al. The Movement disorder society evidence-based medicine review update: Treatments for the motor symptoms of Parkinson's disease. Mov Disord. 2011;26 (Suppl 3):S2-41.

15. Miller KJ, Suárez-Iglesias D, Seijo-Martínez M, Ayán C. Physiotherapy for freezing of gait in Parkinson's disease: a systematic review and meta-analysis. Rev Neurol. 2020; 1;70(5):161-170.

16. Sánchez JL, Buriticá O, Pineda D, Uribe CS, Palacio LG. Prevalence of Parkinson's disease and parkinsonism in a Colombian population using the capture-recapture method. Int J Neurosci. 2004;114(2):175-82.

17. Rossón S, Fuentealba C, Hormazábal C, Villena C, Brieba F. Enfermedad de Parkinson y demencia, calidad de vida y sobrecarga del cuidador. Intervención multidisciplinaria en Atención Primaria. Rev Chilena Salud Publica. 2013. 4;17(1):48-50.

18. Avendaño-Avendaño SB, Bernal-Pacheco O, Esquivia-Pájaro CT. Caracterización funcional y calidad de vida en pacientes con enfermedad de Parkinson. Rev Col Med Fis Reh 2020;2;29(2):93-102.

19. Alejos M, Vázquez-Bourgon J, Santurtún M, Riancho J, Santurtún A. ¿Existe mayor riesgo de suicidio en pacientes diagnosticados de una enfermedad neurológica? Neurologia. 2020;22:S0213-4853(20)30129-8.

20. Miranda MC, Hazard SO, Miranda PV. La música como una herramienta terapéutica en medicina. Rev Chil Neuro-psiquiatr. 2017;55(4):266-77. 


Montserrat Guillermo Durán, es pasante de la licenciatura de fisioterapia y
rehabilitación. Ha sido reconocida como el mejor promedio de su generación,
así como por su desempeño en diversas presentaciones en foros médicos
y congresos a nivel estatal y Latinoamérica. Actualmente se encuentra
realizando su servicio social en el Centro de Enseñanza y Adiestramiento
de Fisioterapia Y Rehabilitación, de la Universidad Marista. Planea especia-
lizarse en la rama geriátrica con especial interés en el manejo de trastornos
neurodegenerativos y en el área de investigación.

